\title{
Three dimension object location based on visual serving
}

\author{
Qinjun $\mathrm{Du}^{\mathrm{a}}$, Shuai $\mathrm{Xu}^{\mathrm{b}}$ and $\mathrm{Hao} \mathrm{Ma}^{\mathrm{c}}$ \\ ${ }^{1}$ School of Electrical and Electronic Engineering, Shandong University of Technology, \\ 12 Zhangzhou road, Shandong Zibo, 255091 China \\ aemail: duqinjun@sdut.edu.cn, bemail:1490801175@qq.com, email: 136115347@qq.com
}

Keywords: three dimension; visual serving; object location.

\begin{abstract}
In the humanoid robot system, the hand-eye system strict significance calibration is very difficult, or even impossible, which greatly increases the difficulty of the humanoid robot for the object manipulation. This paper, based on the visual feedforward and visual feedback control strategy, the humanoid robot completes the operation object. A non strict hand-eye calibration system based on visual feedback is a reasonable way to solve humanoid robot object manipulation. Experiments show that the three-dimensional measurement visual system, based on the visual feedforward and the visual feedback control strategy, improves the accuracy of the location object.
\end{abstract}

\section{Introduction}

Humanoid robot as an intelligent machine which can get to the region to operate object through the body movement and can complete the operation tasks using arm and hand with the help of various sensor information $[1,2]$. In the process of the humanoid robot walk, the joints of the head and the hand will deviate from the standard zero-position; therefore, the humanoid robot hand-eye system strict significance calibration is very difficult, or even impossible, which greatly increases the difficulty of the humanoid robot for the object manipulation [3, 4]. Especially the standard zero-position offset of the head and hand joints have a great impact on the visual servo system of the humanoid robot. Therefore, strict hand-eye system calibration is very difficult for the humanoid robot $[5,6]$.

In this paper, based on the visual feedforward and visual feedback control strategy, the humanoid robot completes the operation object. Using the visual feedforward information, the hand of the humanoid robot approach the target object; using the visual feedback control strategy, the hand of the humanoid robot achieves fine alignment with the target object. A non strict hand-eye calibration system based on visual feedback is used to solve humanoid robot object manipulation.

This paper is organized as follows. The system overview of humanoid robot is described in Section 1. In Section 2, we present the object positioning based on visual servo. In Section 3, the three-dimensional position experiments are provided. Finally, our conclusions are given in Section 4.

\section{The system overview of humanoid robot}

The humanoid robot system is composed of visual information processing system and motion control system; there are two computers responsible respectively, two computers communicate by Memolink which make the two computers share memory information as shown in Fig.1. The visual information processing system is responsible for achieving and processing of the visual information of the target object; the motion control computer is responsible for the movement of each joint of the body, besides control the walk, it needs to control the body balance and the coordination of the hand with foot too. Therefore, the motion control part is a multi-task control system and the control task is very heavy.

The humanoid robot's arm and hand have the same structure and operation functions as the human beings, which can ensure the humanoid robot has the same operating flexibility as the human beings. Therefore, the humanoid robot arm with six degrees of freedom, the hand has three degrees of freedom; in the planning and servo control of the control system, the humanoid robot imitates the human to move and grasp objects within the effective range which the robot arm can reach. 


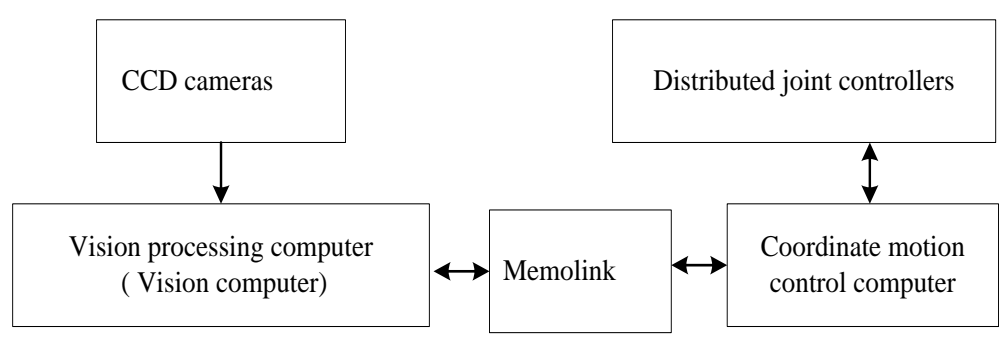

Fig.1. The humanoid robot system architecture

\section{Object positioning based on visual servo}

Humanoid robot binocular stereo vision internal parameters are strictly calibrated; external parameters of the head and arm use the system configuration parameters. In the process of visual servo, the humanoid robot walk close to the target object, head and arm joint null position is relative, always deviate from the ideal model of zero. Therefore, the humanoid robot visual servo is non-strict calibration.

Humanoid robot body structure is a complex system. A red sphere selected as a target, From the point of view of the crawl, the object ball is the same attitude regardless place anyway, the world coordinate system, $O_{0}-X_{0} Y_{0} Z_{0}$ as the goal spherical coordinate system, the hand of the center coordinate $\mathrm{O}_{7}-\mathrm{X}_{7} \mathrm{Y}_{7} \mathrm{Z}_{7}$ with a certain pose.

First calculate the three-dimensional position of the object ball $\left[O_{x}, O_{y}, O_{z}\right]^{T}$, Calculate a desired position of the hand $\left[P_{x}, P_{y}, P_{z}\right]^{T}$, Then set the desired attitude of the hand. Therefore, before grabbing action execution, the desired position and orientation of the humanoid robot hand as the equation (1), where $[\Delta x, \Delta y, \Delta z]^{T}$ is the amount of translation between the target ball coordinates $O_{0}-X_{0} Y_{0} Z_{0}$ and end effector coordinate $\mathrm{O}_{7}-X_{7} Y_{7} Z_{7}$.

$$
T=\left[\begin{array}{cccc}
1 & 0 & 0 & P_{x} \\
0 & 0 & 1 & P_{y} \\
0 & -1 & 0 & P_{z} \\
0 & 0 & 0 & 1
\end{array}\right]=\left[\begin{array}{cccc}
1 & 0 & 0 & O_{x}+\Delta x \\
0 & 0 & 1 & O_{y}+\Delta y \\
0 & -1 & 0 & O_{z}+\Delta z \\
0 & 0 & 0 & 1
\end{array}\right]
$$

The grasping object process of humanoid robot can be divided into five steps: find and coarse positioning target object, walking close to the target object, based on the visual feedforward coarse alignment, fine alignment based on visual feedback, grasp. Each step requires a different control strategy.

In the coarse alignment process of the objectives ball and hand, humanoid robot uses vision-based feedforward control strategy, which is Look-then-Move control mode. Fig.2 shows the control process Look-then-Move. Can be seen from the figure, the entire control process is a serial process, there is no visual feedback is introduced into the robot, and therefore, at this time, the humanoid robot control accuracy will be affected by the accuracy of the robot model accuracy, the accuracy of the model of the camera, the accuracy of the object feature extraction, the accuracy of the pose estimation, any one part of this error could affect the final positioning error. In this paper, the visual feedforward task is to achieve a robot hand and the targeted ball coarse alignment, and to guide hand close to the target object, and to make the hand and the object ball simultaneously appears in the vision of the robot. Based on the visual feedforward control strategy is meeting the requirements of the humanoid robot system.

Assuming that the target location information is $\left[O_{x}, O_{y}, O_{z}\right]^{T}$, the desired position and orientation of the hand is 


$$
T^{*}=\left[\begin{array}{cccc}
1 & 0 & 0 & O_{x}+\Delta x_{1} \\
0 & 0 & 1 & O_{y}+\Delta y_{1} \\
0 & -1 & 0 & O_{z}+\Delta z_{1} \\
0 & 0 & 0 & 1
\end{array}\right]
$$

where $\left(\Delta x_{1}, \Delta y_{1}, \Delta z_{1}\right)$ is the shift amount of the end effector coordinate system relative to the target ball coordinate.

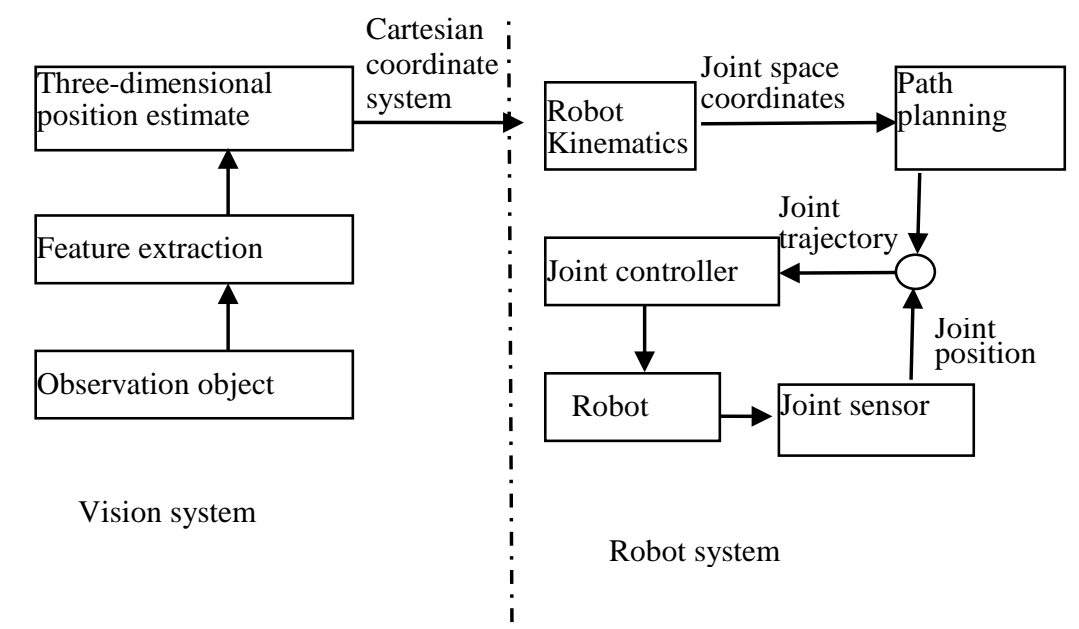

Fig.2 The control process Look-then-Move

In the fine alignment process of the target ball and the hand, we use location-based visual servo control. Based on visual servo position is to use visual feedback to compensate hand-eye model various errors. The task of the visual servo process is described by the kinematics error function $E(x)$ of the current point of the end effector and the destination point. The final goal of the visual servo control is to make the error function satisfies $E\left(x=x^{T}\right)=0$. Where $x(t)$ indicates that the current three-dimensional position information about the hand center, $x^{T}$ indicates the current location information of the target ball. In the alignment process of the hand and the target ball, kinematics errors function as shown in equation (3).

$$
E(x)=x_{T}-x(t)
$$

If using the simple proportional controller ensures error exponentially decline, proportional control as shown in equation (4).

$$
\dot{x}(t)=k \times E(x(t))=k\left(x_{T}-x(t)\right)
$$

In other words, the desired speed of movement of the hand is proportional to the distance between the hand and the target object.

\section{Three dimension object location experiments}

We use the motion capture system to measure the three-dimensional position of the target object, the three-dimensional position of the target object as an absolute spatial position, as a benchmark to measure the measurement accuracy of the humanoid robot vision platform. In the experiment, red target ball is placed in the scene.

Fig.3 (a) is the X coordinate measurements in the world coordinate system, experiments show that in the $\mathrm{x}$ direction of the world coordinate system, the target ball and hand measurements to some extent deviated from the position of absolute space, but the space distance just as the absolute space distance, the error is less than $1 \%$.

Fig.3 (b) Y coordinate measurements in the world coordinate system , the target ball and hand visual measurements are stable, to some extent a deviation from absolute coordinates, but the distance just as the absolute space, the distance errors less than $2 \%$. 
Fig.3 (c) shows the measurement results in the $\mathrm{z}$ direction in the world coordinate system, the data show that in the $\mathrm{z}$ direction of the world coordinate system of the target object and hand measurements are certain deviations from the absolute coordinate extent, but the distance and the absolute space between consistent distance errors of less than $2 \%$.

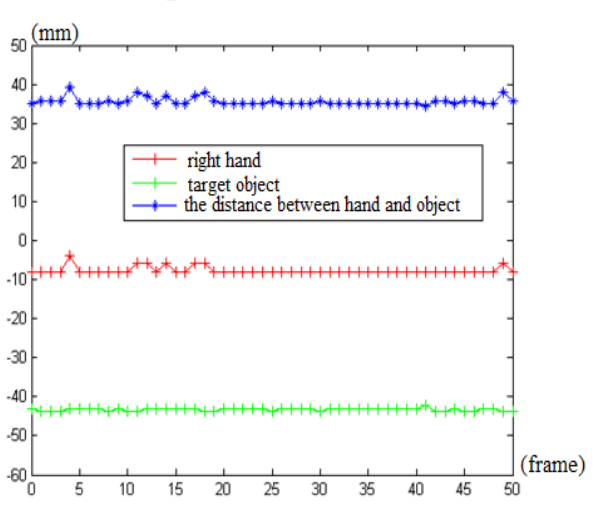

(a)

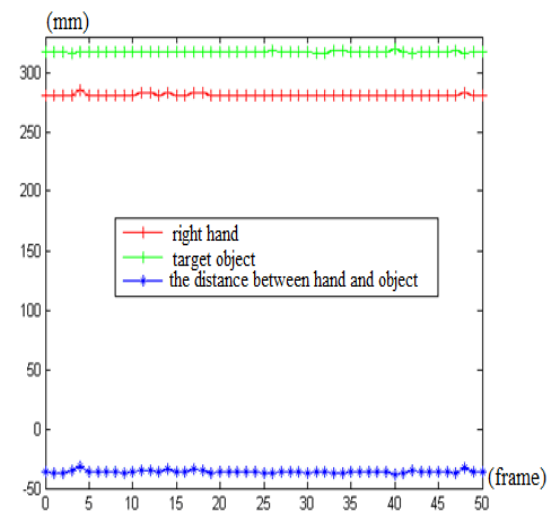

(b)

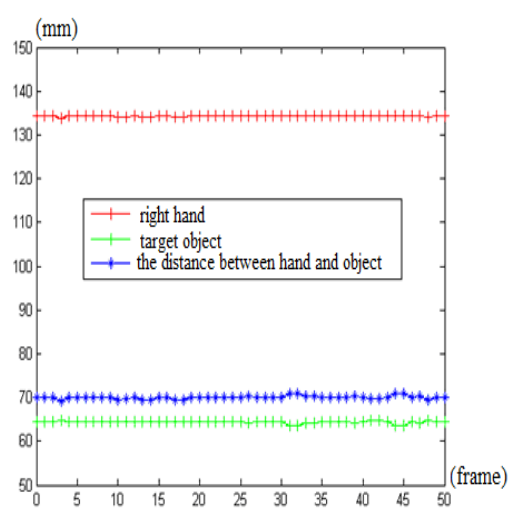

(c)

Fig.3. X Y Z coordinate measurements in the world coordinate system

\section{Summary}

This paper analyzes the non-strict calibration humanoid robot vision positioning system. In view of non-strict calibration humanoid robot hand-eye system, we put forward the object position strategy, Experiments show that the three-dimensional measurements of the visual system to spatial points deviate from the absolute spatial position to a certain extent, but system errors almost cancel each other out, the three-dimensional distance between the target object and the hand just the same as the motion capture systems, the accuracy is meeting the humanoid robot system requirement.

\section{Acknowledgements}

This work was financially supported by the National Natural Science Foundation of China (61175090).

\section{References}

[1] Young S.C., Kang G.K., Ji Y.L., et al. MAHRU-M: A mobile humanoid robot platform based on a dual-network control system and coordinated task execution. Robotics and Autonomous Systems, Vol.59, No.6 (2011), pp. 354-366.

[2] Browatzki, Bjorn; Tikhanoff, Vadim; Metta, Giorgio; et al. Active in-hand object recognition on a humanoid robot, IEEE Transactions on Robotics, Vol.30, No.5, pp. 1260-1269, October 1, 2014.

[3] Kang, Tae-Koo; Lim, Myo-Taeg; Park, Gwi-Tae; et al. 3D vision-based local path planning system of a humanoid robot for obstacle avoidance, Journal of Electrical Engineering and Technology, Vol.8, No.4, pp. 879-888, July 2013.

[4] Jia D.Y., Huang Q., Tian Y., et al. (2009) Object manipulatin of a humanoid robot based on visual feedforward and visual feedback. Transactions of Beijing Institute of Technology, Vol.29, No.11, pp. 983-987.

[5] Du Q.J., Shi X., Dai B., et al. Binocular stereo vision system for a humanoid robot, International Journal of Computer Applications in Technology, Vol. 46, No. 4(2013). pp. 316-322.

[6] G. Chesi, K. Hashimoto. Effects of camera calibration errors on static-eye and hand-eye visual servoing. Advanced Robotics, 2003, 17(10):1023-1039. 